\title{
Anti-Reciprocal Motional Impedance and its Properties
}

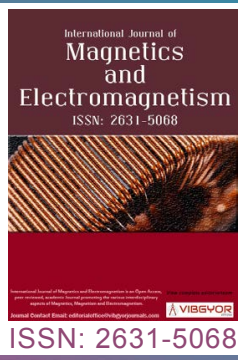

\section{Noori Kim ${ }^{1^{*}}$ and Jont B Allen ${ }^{2}$}

\section{${ }^{1}$ Electrical and Computer Engineering Department, DigiPen Institute of Technology, Singapore \\ ${ }^{2}$ Electrical and Computer Engineering Department, University of Illinois Urbana-Champaign, USA}

\begin{abstract}
We investigate a classical notion denoted as 'Motional impedance $\left(Z_{\text {mot }}\right)$ ' first developed by Kennelly in 1912 , then explored by many other researchers early in the $20^{\text {th }}$ century. Due to long history to establish impedance properties, $Z_{\text {mot }}$ has not been clearly understood with respect to classical impedance properties which makes retroactive study of the $Z_{\text {mot }}$ worthy. In this study, we project $Z_{\text {mot }}$ 's unique characteristics onto anti-reciprocity where every electro-mechanical system as modeled by a gyrator. This work also clarifies that $Z_{\text {mot }}$ is a type of transfer impedance, thus is not necessarily to be a minimum-phase nor a Positive-Real function. Based on simulation, this study shows a shunt eddy current loss on the electrical side of the system is one possible source of the negative real part of $Z_{\text {mot }}$ which leads negative real part in $Z_{\text {mot }}$ plots. By taking a Balance Armature Receiver (BAR) as a specific example of the electro-mechanical systems, we also explore the (non)linear approximation principal of the BAR based on the magnetic force and hysteresis characteristics. We believe that this study puts anti-reciprocal physics on both the empirical and theoretical basis.
\end{abstract}

\section{Keywords}

Motional impedance, Anti-reciprocal systems

\section{Introduction and Background}

The concept of motional impedance $\left(Z_{\text {mot }}\right)$ was first suggested by Arthur E. Kennelly in $1912^{\mathrm{a}}$. It is a unique characteristic of every electro-mechanical

${ }^{a}$ Kennelly was born in 1861 in India and worked with Edison starting in 1887 at the age of 26 years. He then became a professor of electrical engineering at both Harvard University and the Massachusetts Institute of Technology (MIT). system such as earphones, loudspeakers, hybrid cars, etc. The $Z_{\text {mot }}$ is defined as the component of the electrical impedance proportional to the output velocity. From measurements of the electrical impedance at the electrical input, one may accurately estimate the mechanical or acoustic load impedance. This property alone makes it worth of study [1].

In 1827, Georg Ohm generalized measurement relationship between applied voltage and current

*Corresponding author: Noori Kim, Electrical and Computer Engineering Department, DigiPen Institute of Technology, S 139660, Singapore

Accepted: June 13, 2019; Published: June 15, 2019

Copyright: (C) $2019 \mathrm{Kim} \mathrm{N}$, et al. This is an open-access article distributed under the terms of the Creative Commons Attribution License, which permits unrestricted use, distribution, and reproduction in any medium, provided the original author and source are credited.

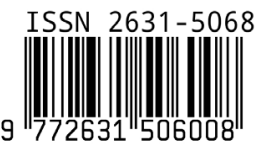

Kim and Allen. Int J Magnetics Electromagnetism 2019, 5:021 
varying resistance (as known as Ohm's law suggested only for DC circuits initially). Kennelly was the first person to coin the term as impedance for alternating current (AC) circuits [2] ${ }^{\mathrm{b}}$ and the first to utilize complex numbers in the circuit theory. Campbell's work follows the work of Heaviside [3] who first suggested loaded lines which leads to the generalized wave filter invention based on quasistatic (QS) theory [4].

In 1931, Brune first characterized a driving-point impedance as a positive-real (PR) quantity (positive definite operator in matrix form), requiring that every physically realizable impedance cannot have a negative resistance [5]. Brune's impedance is consistently studied with classic circuit theories such as Kirchhoff's circuit laws (KCL and KVL) under the quasistatic (QS) condition. The $Q S$ assumption requires no system delay (only bandlimited delay). Every QS impedance network is represented using lumped circuit elements such as resistors, inductors, and capacitors [6]. All Brune PR impedances are minimum phase (MP), because every PR function must be MP. Thus, every Brune impedance is $P R, M P$, and $Q S$ [7].

Kennelly defined $Z_{\text {mot }}$ as the difference between the two driving-point impedances,

$$
Z_{\text {mot }}=\left.Z_{\text {in }}\right|_{\text {free }}-\left.Z_{\text {in }}\right|_{\text {blocked }} \text {. }
$$

Kennelly and Affel in 1915 published a second paper where $Z_{\text {mot }}$ is characterized as a circle passing through the origin, with its diameter depressed by a certain angle (depressed compared to the circle in undamped impedance) in the impedance plane $(Z(\omega)=R(\omega)+j l(\omega))$. Kennelly and Affel described the circle in terms of the electrical and mechanical properties of the system. However, no justification for why the $Z_{\text {mot }}(\omega)$ subscribed a circle was given. In 1954 Hunt further discusses the circle feature, again with no rationale for this functional form.

Kennelly's third paper about $Z_{\text {mot }}$ [8] was focused on power concept of $Z_{\text {mot' }}$ and introduced motional power diagram, to provide a better physical un-

${ }^{\mathrm{b}}$ The properties of the impedance have been established over a long-time span, Ohm (1827, DC impedance) $\rightarrow$ Kennelly (1893, AC impedance) $\rightarrow$ Brune (1931, establishment of impedance properties). Note that Heaviside [3] defined the term 'impedance' in 1982, a few months later in April 1893, Kennelly extended the definition of impedance to AC circuits [2]. derstanding of the $Z_{\text {mot }}$ circle (in their view, power seemed a better concept to understand the system, compared to impedance). The motional power diagram is drawn based on magnetomotive force generated by the vibration of the diaphragm in the permanent magnetic field. They explained the motional power circle by means of 'active mechanical power', which is defined as a difference between electrical power and hysteresis power.

In 1921, he went on to describe some techniques to measure various acoustic constants introduced in his previous three papers [9]. Historically, this work can be viewed as the first mechanical/acoustic impedance calibration purely from the electrical measurements. This idea was extended to compute acoustic impedance based on purely electrical data [10]. Given the practical importance of this technique, it needed to be fully characterized. This method is discussed in Ramo, et al. [1].

Besides Kennelly, Wegel [11] also considered $Z_{\text {mot }}$. This paper is credited by Hunt as the inspiration of Hunt's 1954 two-port matrix representation. Wegel takes account of the general theory of receiver structures using a simple schematic having four coils. As applications, he takes four different specific cases of a receiver: A simple receiver, a receiver with eddy currents in the core, a simple induction-type receiver, and an electrodynamic receiver. He describes the receiver eddy current, which decreases proportional to square root of the frequency due to the diffusion and magnetic flux into the conducting iron core. However, the author did not derive any specific formula for this phenomenon, as it was simply an experimental observation. Vanderkooy [12] has defined the eddy-current formula from Maxwell's equation. He demonstrated that the eddy-current is a secondary current flow generated from the primary magnetic field.

Frederick Vinton Hunt, an acoustic engineer, was influenced by Kennelly's discoveries. Hunt published Electroacoustics in 1954, in which, he analyzed and synthesized the electroacoustic (or electromechanical) system, modeling it as a $2 \times 2$ matrix using scalar forms of Ampere's law and Far-

'It was done by distinguishing two constants $\mathrm{k}=\sqrt{-1}$ for a $90^{\circ}$ time phase shift and $\mathrm{k}=\sqrt{-1}$ for a $90^{\circ}$ spatial phase shift [17]. $F=B l k l$ and $\phi=B l k u$, where $F, I, \phi, u, B, l$ are force, current, voltage, velocity, magnetic intensity, and length of wire, respectively. 
aday's law'. Hunt's two-port matrix parameters can be modeled using transmission and impedance matrix methods [7].

Understanding that definition of an "impedance" is a ratio between potential and flow of a field (i.e., electrical, acoustic, or mechanical fields), one can expand the definition of impedance in multi-port network systems such as electromagnetic, electroacoustic and etc. Therefore, other than the classical driving point impedance, transfer impedance (i.e., voltage over velocity, current over velocity, or force over current and etc) was naturally developed, defined, and used by researchers to critically analyze and understand multidisciplinary systems.

The $Z_{\text {mot }}$ is a kind of transfer impedance. Retroactively applying Brune's work, therefore $Z_{\text {mot }}$ does not obey the PR property. However, to our knowledge, characteristics of the $Z_{\text {mot }}$ such as its negative real parts and circular shape, have yet to be clarified with regard to its physical properties [13-15]. Therefore, we aim to access the $Z_{\text {mot }}$ given its historical, conceptual, and physical analysis, and simulation. To engage in this goal, we will reduce the complexity of the proposed BAR model [16] to its essential elements for simplifying simulation purpose.

The remainder of this study is structured as follows: Section 2 introduces theoretical concepts with definitions of $Z_{\text {mot }}$ as defined by Kennelly and Hunt. Section 3 provides a simple electro-magnetic model. To make use of the model, the result of the simulation follows in Section 4. Finally, we explain a possible physical reasons to make the negative real parts and the circle shape of the $Z_{\text {mot }}$.

\section{Methods}

In this section, we review important theories needed to understand $Z_{\text {mot }}$ and anti-reciprocal networks. These include the definition and essential properties of Hunt's two-port equations which are required to define $Z_{\text {mot }}$

\section{Definition of motional impedance $\left(Z_{\text {mot }}\right)$}

Following Wegel [11], Hunt [17] modeled an electro-mechanic system into a simple $2 \times 2$ impedance matrix relationship. There are four two-port network parameters, $Z_{e}(s), Z_{m}(s), T_{e m}(s)$, and $T_{m e}(s)$ :

$$
\left[\begin{array}{l}
\Phi(\omega) \\
F(\omega)
\end{array}\right]=\left[\begin{array}{cc}
Z_{e}(s) & T_{e m}(s) \\
T_{m e}(s) & Z_{m}(s)
\end{array}\right]\left[\begin{array}{c}
I(\omega) \\
U(\omega)
\end{array}\right],
$$

$$
\begin{aligned}
& Z_{e}=\frac{\Phi}{I} \text { when } \mathrm{U}=0, \\
& T_{e m}=\frac{\Phi}{U} \text { when } \mathrm{I}=0, \\
& T_{m e}=\frac{F}{I} \text { when } \mathrm{U}=0, \\
& Z_{m}=\frac{F}{U} \text { when } \mathrm{I}=0,
\end{aligned}
$$

Where $\Phi, I, F$, and $U$ are signals, the voltage, current, force, and velocity in frequency domain respectively, and ' $s=\sigma+j \omega$ ' is the Laplace frequency [16].

Along with Eq. 2, the two-port 'electro-mechanic' transducer equation can alternatively be represented in $A B C D$ matrix form, as given by

$$
\left[\begin{array}{c}
\Phi(\omega) \\
I(\omega)
\end{array}\right]=\left[\begin{array}{ll}
A(s) & B(s) \\
C(s) & D(s)
\end{array}\right]\left[\begin{array}{c}
F(\omega) \\
-U(\omega)
\end{array}\right] .
$$

Conversion between Eq. 7 and Eq. 2 has following relationship

$$
\left[\begin{array}{ll}
A(s) & B(s) \\
C(s) & D(s)
\end{array}\right]=\frac{1}{T_{m e}(s)}\left[\begin{array}{cc}
Z_{e}(s) & \Delta_{z} \\
1 & Z_{m}(s)
\end{array}\right]
$$

Where $\Delta_{z}=Z_{e} Z_{m}-T_{e m} T_{m e}$. Note that $\Delta_{z}= \pm 1$ for a reciprocal and an anti-reciprocal systems respectively. For the impedance matrix to exist $C=\frac{1}{T_{m e}} \neq 0$, which means that $T_{m e}$ cannot be $\infty$.

When the force ' $F$ ' is zero in Eq. 2,

$$
\frac{\Phi}{I}=Z_{e}+\frac{T_{e m} U}{I}
$$

and

$$
\frac{U}{I}=-\frac{T_{m e}}{Z_{m}} \text {. }
$$

Thus the 'unconstrained' electrical input impedance is

$\left.Z_{\text {in }}\right|_{F=0}=\left.\frac{\Phi}{I}\right|_{F=0}=Z_{e}-\frac{T_{e m} T_{m e}}{Z_{m}}=Z_{e}+Z_{m o t}$.

The $Z_{\text {mot }}$ is defined as the difference between two mechanical boundary conditions on the electrical impedance $\left(Z_{\text {in }}\right)^{\text {d: }}$ 1) $Z_{\text {in }}$ with freely oscillating

'The electrical conditions, 'open' and 'short', are synonymous with the two mechanical terms, 'blocked' and 'free (unconstrained)', respectively. 
(vibrating) mechanical side $(F=0$ : Unconstrained condition),

2) $Z_{\text {in }}$ with the mechanical system is not allowed to move (Ze, $U=0$ : Blocked condition, Eq. 3),

$Z_{m o t}=-\frac{T_{e m} T_{m e}}{Z_{m}}=-T_{e m} T_{m e} Y_{m}=\left.Z_{i n}\right|_{F=0}-Z_{e}=\left.Z_{i n}\right|_{F=0}-\left.Z_{i n}\right|_{U=0}$.

Manipulating Eq. 8, Eq. 11 becomes

$$
Z_{m o t}=\frac{\Delta_{z}}{Z_{m}}-Z_{e}=-Y_{m}-Z_{e},
$$

Since $\Delta_{z}=-1$ for the anti-reciprocal system. Equation 11 and 12 reinforce that $Z_{\text {mot }}$ does not have to be a Minimum-Phase (MP) nor PR function as the off-diagonal elements of Hunt's matrix, $T_{e m}$ and $T_{m e^{\prime}}$ can augment delay lines. Also a difference between two PR functions does not have to be a PR function. More about these postulates follow next section.

\section{Core losses in EM transducer: Eddy current loss and Hysteresis loss}

Starting from Maxwell's equations we derive the core losses of motors by the use of Poynting's theorem (neglecting the source charge density and displacement current terms). By manipulating one of vector identities one may calculate the energy flow (W) into the volume (V) between two time points $(t 1, t 2)$,

$$
W=-\int_{t 1}^{12} \int_{V}[\mathbf{E}(\mathrm{t}) \times \mathbf{H}(\mathrm{t})] \cdot \mathbf{d S d t}=\int_{t i}^{12} \int_{V}^{1}\left[\mathbf{H}(\mathrm{t}) \cdot \frac{\mathbf{d B}(\mathrm{t})}{\mathbf{d t}}+\mathrm{E}(\mathrm{t}) \cdot \underset{\nabla \times \mathbf{H}}{\mathbf{J}(\mathbf{t})}\right] \mathbf{d V d t} .
$$

Here the displacement current term may be ignored for a conducting medium (i.e., conductance of a material, $\sigma \neq 0$ ), leaving only $\mathrm{J}(\mathrm{t})^{\mathrm{e}}$. For example, one can calculate a propagation cutoff frequency of two waves (diffusion and normal) in a medium, set the two terms in equal to each other. If

$$
\begin{aligned}
& \underbrace{\mu \sigma \frac{\partial \mathbf{H}}{\partial t}}_{\text {diffusion }}=\underbrace{\mu \in \frac{\partial^{2} \mathbf{H}}{\partial t^{2}}}_{\text {normal }}, \\
& \mu \sigma(j \omega) \mathbf{H}=\mu \in(j \omega)^{2} \mathbf{H}, \\
& \sigma=\epsilon(j \omega),
\end{aligned}
$$

the cutoff frequency $\left(f_{c}\right)$ is

$$
f_{c}=\frac{\sigma}{2 \pi \epsilon} \text {. }
$$

\footnotetext{
$\nabla \times \mathbf{H}(t)=\sigma \mathbf{E}(t)+\frac{\partial \mathrm{D}(t)^{0}}{\partial t}=\mathrm{J}(t)$.
}

The $f_{c}$ of copper, for example, is about 4300 $[\mathrm{GHz}]\left(\sigma=5.96 \times 10^{7}, \epsilon_{\mathrm{r}}=250,000, \epsilon_{0}=8.854 \times 10^{-}\right.$ $\left.{ }^{12}\right)$, meaning that the wave below this frequency is diffusive. The corresponding wave length $\left(\lambda_{c}\right)$ can be calculated as

$$
\lambda_{c}=\frac{c_{\text {copper }}}{f_{c}}=\frac{3 \times 10^{8}}{4.3 \times 10^{12} \sqrt{250,000}} \approx 0.14 \mu[\mathrm{m}],
$$

Where $c_{\text {copper }}=\frac{c_{0}}{\sqrt{\epsilon_{r}}}$.

As the material is homogeneous, Eq. 13 reduces to

$$
W=\int_{V}[\underbrace{\int_{B 1}^{B 2} H d B}_{\text {Hysteresis loss }}+\underbrace{\frac{1}{\sigma} \int_{t 1}^{t 2} J^{2}(t) d t}_{\text {Eddy current loss }}] d V,
$$

Where $B 1$ and $B 2$ are the flux densities associated with $t 1$ and $t 2$, respectively. When the current (i.e., current density $\mathrm{J}$ ) is generated from the system source, it induces the magnetic field (H) which results in two losses (Eq. 13). The air and the armature share the same induced $\mathbf{H}$; however, due to the permeability differences between two mediums $\left(\mu_{0}=4 \pi \times 10^{-7}\right.$ and $\mu_{i} \approx 2.5 \times 10^{-1}$ for the vacuum and iron, respectively), the effective magnetic densities $(\mathbf{B}=\mu \mathrm{H})$ of each medium are different by a factor of $2 \times 10^{5}$.

To increase the coupling magnetic force in Eq. 20 , one can choose a softer material (that means choose the material having a smaller $\mu$ ) to minimize the hysteresis loss in the system.

$$
f_{\text {mag }}=\frac{\Psi^{2}}{2 \mu_{i} A},
$$

Where $\Psi$ is the magnetic flux defined as B times the effective area (A) [17]. Equation 20 comes from the Maxwell stress tensor. Notice that Eq. 20 goes as $\Psi^{2}$ which leads a non-linear behavior in electromagnetic systems $[17,18]$. In case of a BAR, $\Psi$ in Eq. 20 can be expressed as a sum of $\Psi_{D C}$ and $\Psi_{A C}$.

$$
f_{\text {mag }}=\frac{1}{2 \mu_{i} A}(\underbrace{\Psi_{D C}^{2}}_{i)}+\underbrace{2 \Psi_{D C} \Psi_{A C}}_{i i)}+\underbrace{\Psi_{A C}^{2}}_{i i i)}) \text {, }
$$

Where $\Psi_{D C}$ and $\Psi_{A C}$ are fluxes due to permanent magnets and a coil respectively. The i) Term in Eq. 21 is the steady pull on the armature which results in balancing the armature at the pivot. Assuming the $\Psi_{A C}$ to be sinusoidal, the iii) Term gives rise to a second harmonic force on the system with a negligible effect. Therefore, the remaining ii) Term takes 


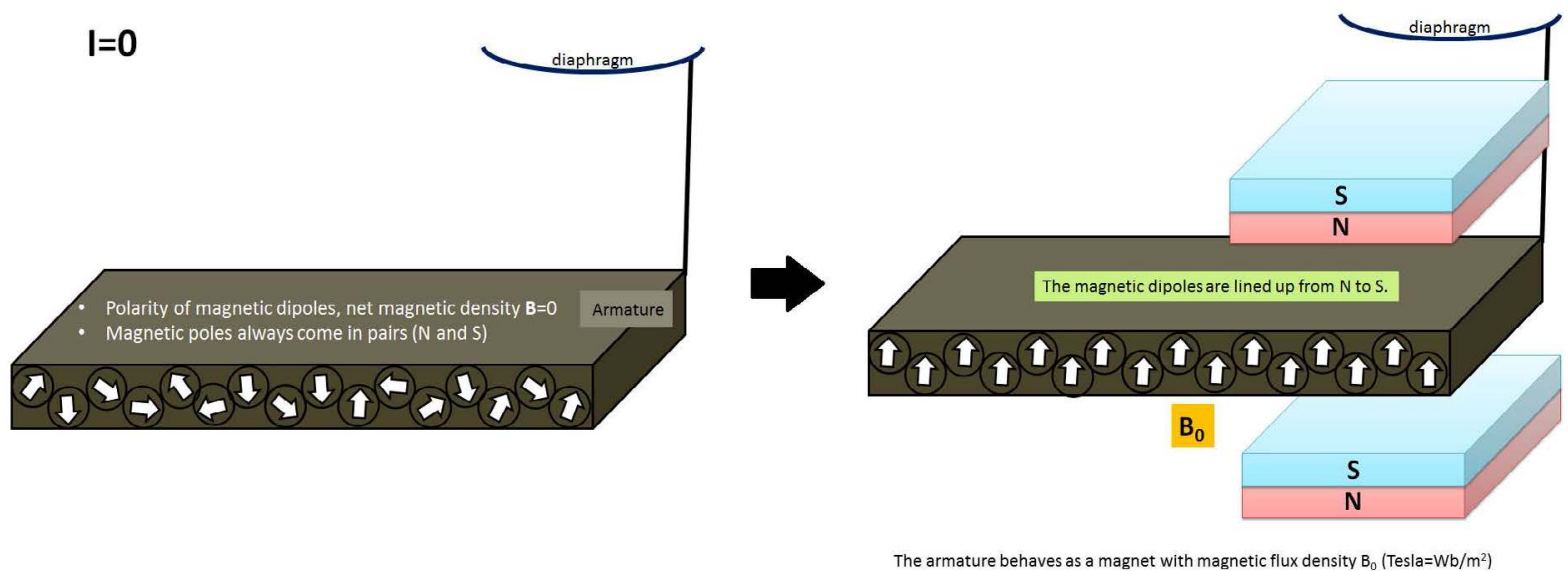

Figure 1: This figure explains the polarity changes of the magnetic dipoles of the armature in DC status (no current). The armature is made by ferromagnetic material, without exposing to magnets it keeps neutral status, the net magnetic density $B$ is zero. Note that unlike a charge, magnetic poles always come in pairs ( $\mathrm{N}$ and S). However, once it exposes to permanent magnets, the armature behaves as a magnet with magnetic flux density $B_{0}$ with a unit of Tesla $=W b / \mathrm{m}^{2}$.

the principal role of $f_{\text {mag }}$, so that the force can be linearly approximated. To investigate the hysteresis characteristic (a.k.a. B and $\mathbf{H}$ curve) of the BAR, its operational path may be drawn as a small lens shape on the full hysteresis loop of the ferromagnetic material (i.e., armature) [17]. By approximating this elliptic shape as a linear line, one assumes a constant permeability which may bring computational benefits. The area of the lens corresponds to a hysteresis loss term, due to the movement of the magnetically polarized domains, in response to the magnetic field (i.e., Force $\propto$ flux ${ }^{2}$ ).

To reduce the eddy current loss, one can consider the skin depth $(\delta)$ of the material which is inversely exponentially proportional to the eddy current (meaning that the strength of the eddy current is stronger on the surface and exponentially weaker in the core of the material),

$$
\delta=\sqrt{\frac{2}{\omega \sigma \mu}},
$$

Where $\omega$ and $\sigma$ are the angular frequency and conductivity of the material [12]. The strength of eddy current becomes smaller with distance from the coil and its density attenuates exponentially with the skin depth. Therefore, choosing a hard material (large in its $\mu$ ) reduces the eddy current loss. This is the opposite of the case of the hysteresis loss. Based on Eq. 20, if we choose a hard material, we expect weaker coupling magnetic force across the system.

\section{Simplification and Simulation of the Bal- anced Armature Receiver model}

For the further application, we introduce a simple electro-mechanic network model including a semi-inductor. The goal here is to demonstrate some condition that $\mathrm{RZ} Z_{\text {mot }}<0$ based on the simplified electro-mechanic model. To suggest a simplest possible electro-magnetic model with the physical explanation, we take an example of the BAR transducer.

Figure 1 explains DC status of the BAR considering only the armature and two magnets. Assuming that the armature consists of many magnetic dipoles, the initial net polarity of the armature without magnets is neutral. However, the dipoles line up from north to south once it is influenced by permanent magnets, and the armature behaves like a magnet with $\mathrm{DC}$ magnetic density $B_{0}$.

More details about BAR operation principle are illustrated in Figure 2; the BAR's behavior when I = 0 and $I \neq 0$. Not shown in this figure, however, when there is a current $(I \neq 0)$ in the coil which surrounds the armature, there is an eddy current which does not couple to the armature's movement. When there is no current in the coil (left of Figure 2), the armature is balanced ${ }^{f}$, so all fluxes and forces are

The name of the BAR, balanced armature receiver, is originated from this $\mathrm{DC}$ status of the device. 

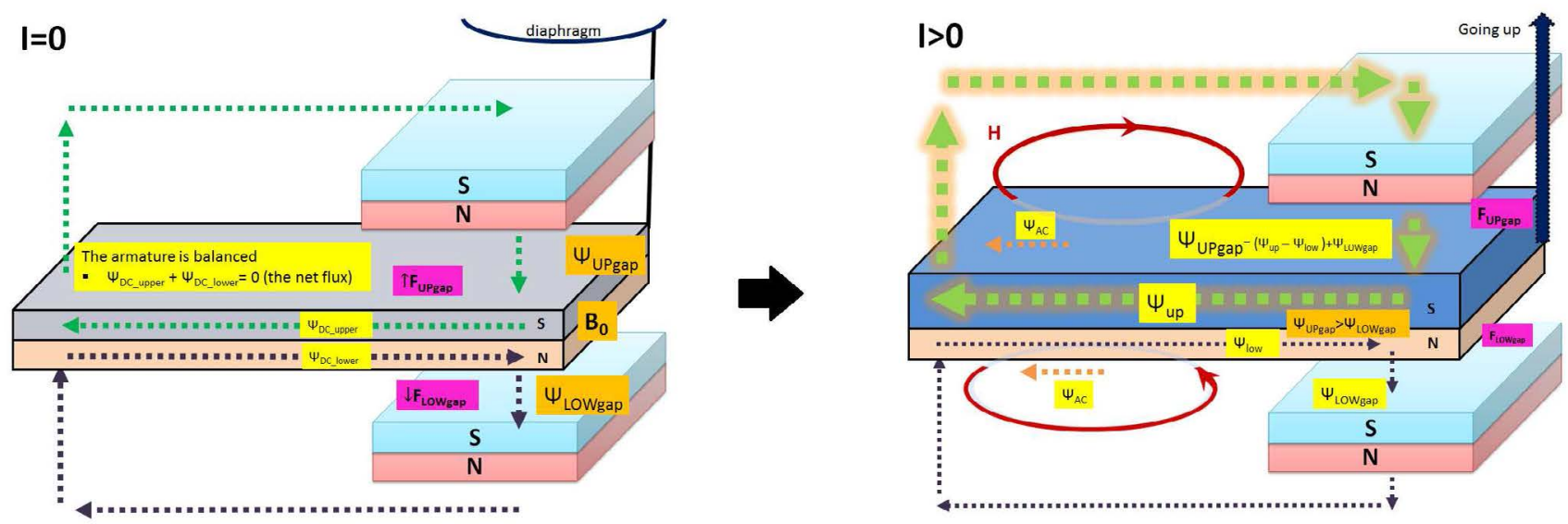

Figure 2: BAR's operational principle via illustration. The left figure shows the case when current (I) is zero, DC status. Due to the permanent magnet, the armature acts as like a magnet. One can draw two flux loops for the upper side of the armature $\left(\Psi_{\mathrm{DC} \text {-upper }}\right)$ and the lower side of the armature $\Psi_{\mathrm{DC} \text {-lower }}$. When there is no current, the armature is balanced, not moving. The right figure shows the case when the current is not zero. Specifically, we take a case when current is greater zero. Due to the AC magnetic flux $\left(\Psi_{A C}\right)$ generated via conducting current from the coil, the size of upper flux loop $\left(\Psi_{\text {up }}\right)$ is now larger than that of lower flux loop $\left(\Psi_{\text {low }}\right)$. AS a result, the magnetic force for the upper gap is greater $\left(F_{\text {Upgap }}\right)$ than the lower gap $\left(F_{\text {Lowgap }}\right)$, the armature goes up, is moving.

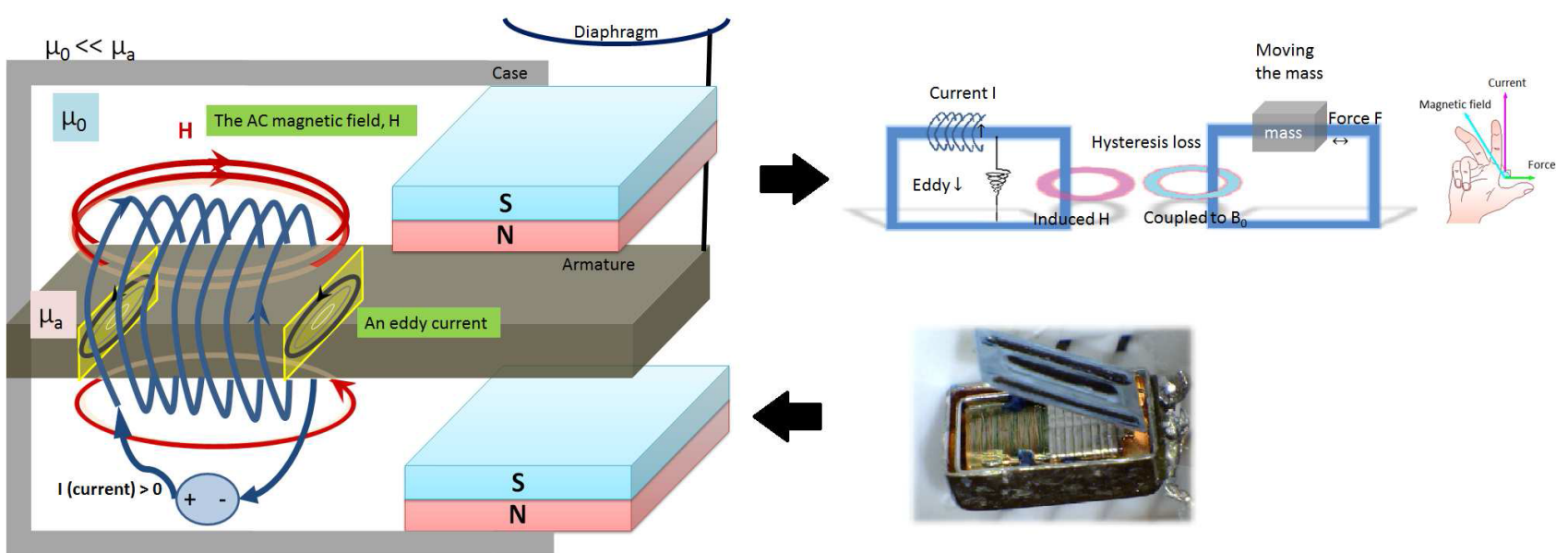

Figure 3: This figure shows the schematic modeling process of the BAR (Knowles ED7045) starting from the real picture of the device after detaching its front case (right bottom) to show its internal components. Core physical components of a BAR include a diaphragm, a coil, an armature, magnets, and case which give rise to current (I), magnetic field $(H)$, eddy current, hysteresis loss, force $(F)$, and etc. The left is the schematic representation of the BAR. The right top figure is the 3D model of BAR which consists of essential elements of the device; an inductor, a semi-inductor, a gyrator and mass. Note directions of the variables (F, H, and I) follow Fleming's right-hand rule.

also balanced. When current flows through the coil, the armature vibrates based on the direction of the AC current. Let us take the case when the current is greater than zero as depicted in the right part of Figure 2. In this case, the flux on the upper part of the armature, $\Psi_{u p}$, becomes a sum of $\Psi_{D C}$-upper and $\Psi_{A C}$ and the flux on the lower part of the armature, $\Psi_{\text {low' }}$, becomes a difference of those two fluxes so that $\Psi_{u p}$ is larger than $\Psi_{\text {low }}$ in this case. Therefore, one can draw relatively greater flux loop on the upper part of the BAR than the lower part so that the magnetic force (Eq. 20) of the upper part is larger to make the armature go up.

Finally, Figure 3 shows the simplifying process of modeling the BAR to help conceptual understanding, starting from the picture (the right bot- 

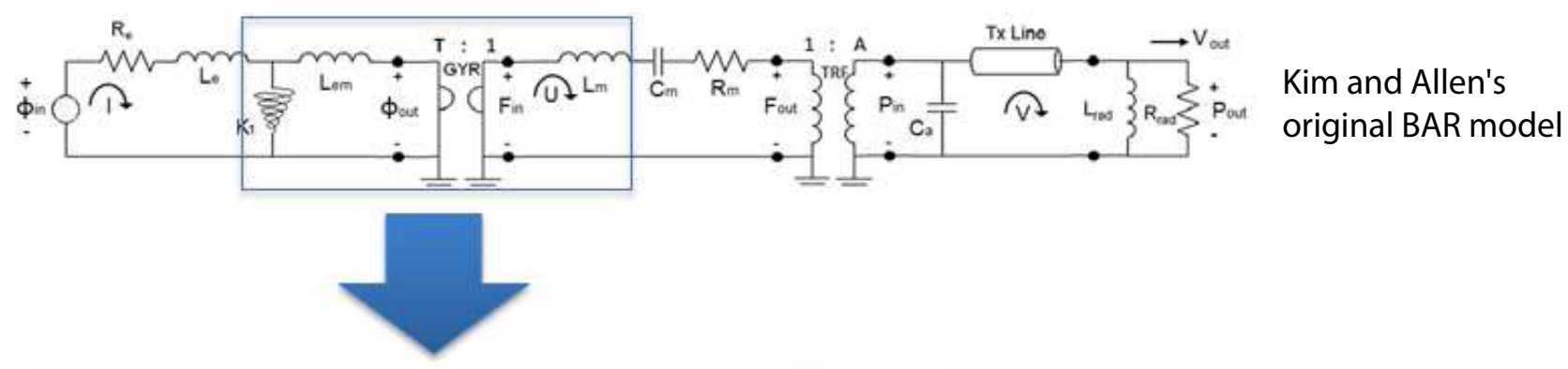

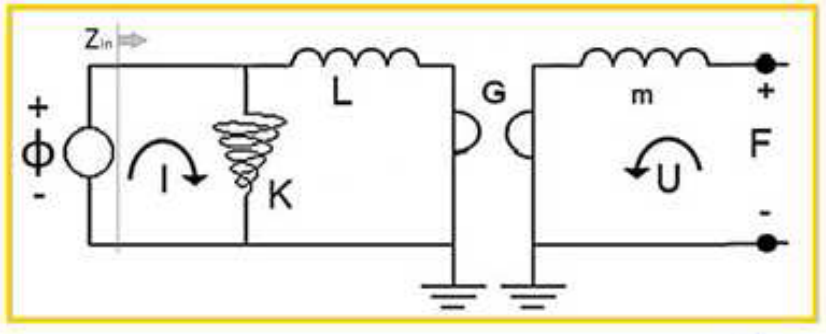

[Short] $Z_{\text {in }}|\mathrm{F}=0=K \sqrt{s}| \mid\left(\mathrm{LS}+\frac{1}{\mathrm{~ms}}\right)$

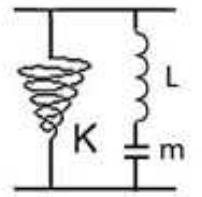

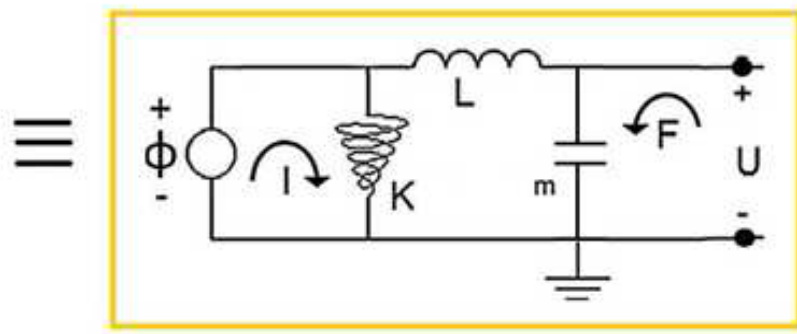

[Open] $\left.Z_{n}\right|_{U=0}=K \sqrt{s}||$ LS

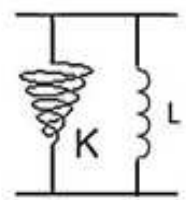

Figure 4: The top figure shows the Balanced Armature Receiver (BAR) circuit model from Kim and Allen [16]. The electrical and the mechanical circuits are coupled by a gyrator (GYR, realizing an anti-reciprocal network), while a transformer (TRF) is used for the coupling of the mechanical and the acoustical circuits. The $K 1$ is a semiinductor representing 'electro-magnetic diffusion' due to 'skin effect'. And the TX Line stands for a transmission line to involve delay in the system, breaking a quasistatic assumption in this electro-acoustic system. This non quasistatic element is the proper way of modeling this system. In this full model, the input and output potentials for each section are specified as voltage $(\Phi)$, force $(F)$, and pressure $(P)$. Current $(I)$, particle velocity (U), and volume velocity $(\mathrm{V})$ represent the flow for each of the three physical sections. The lower left circuit: A simple anti-reciprocal network with a semi-inductor presence. The lower right circuit: The dual representation of the left circuit (equivalent) by applying mobility analogy beyond the gyrator. $Z_{\text {mot }}$ is reconsidered based on Eq. 11 . The frequency dependent real parts (shunt loss) of the semi-inductor in $Z_{\text {in } \mid F=0}$ (short) experience positive phase shift when the open condition impedance $\left(Z_{i n / U=0}\right)$ is subtracted from it.

tom picture, Knowles ED7045). Illustrative representation of the picture is shown on the left part of the Figure 3: A coil is surrounding the armature in the middle of the device. When there is a current flow (I), it generates the magnetic field $(\mathbf{H})$. There is an eddy current loss when the induced $\mathrm{H}$ couples to the armature. A part of $\mathrm{H}$ penetrates into the armature based to its skin depth generating eddy currents which direction is opposite to the the conducting current. This phenomenon is independent of the permanent magnets meaning that the eddy current cannot be seen from the mechanical side of the device. The armature behaves like a magnet due to the permanent magnets sandwiching the armature as shown in Figure 1. It alters its polarity to vibrate the armature based on the direction of the AC current. During the polarity changing process, this system experiences hysteresis loss, the energy required to rotate the domains of magnetic dipoles in the armature. Note that the magnetic density of armature is defined based on the permeability of armature $\left(B=\mu_{\mathrm{a}} H\right)$ which is much greater than the permeability of air $\left(\mu_{0}\right)$. As the diaphragm is attached to the tip of the armature, when the armature moves, the diaphragm also moves.

The simple electro-mechanic model has been reduced from the Kim and Allen's original work (Figure 4: The electro-acoustic network model, Kim and Allen [16]. Related theories are discussed in section 2.1 . 
Left sided figure in Figure 4 shows a oversimplified two-port network containing only essential components for better and easier understanding of the physical electro-mechanic system. In this simple model, any acoustic or resistive components are eliminated.

In this figure we have four components: A semiinductor, an inductor in the electrical port, a mass in the mechanical port, and a gyrator that links two ports.

The two circuits in Figure 4 represent equivalent circuits via the mobility (dual) analogy. In both, very low and high frequencies the capacitor ' $\mathrm{m}$ ' is opened. The parallel relation of semi-inductor and inductor enables the semi-inductor's high frequency dominance Vanderkooy [12]. The mid frequen$c y$ is governed by the inductor $L$ and the capacitor $\mathrm{m}$. If we ignore the semi-inductor in Figure 4, the system looks like a Helmhorltz resonator with neck mass $\mathbf{L}$ and barrel compliance $m$. Therefore, these two components act like a resonator in the system.

To realize this system into a matrix form, we can use $A B C D$ matrix cascading method which results in Eq. 23.

$\left[\begin{array}{c}\Phi(\omega) \\ I(\omega)\end{array}\right]=\left[\begin{array}{cc}1 & 0 \\ \frac{1}{k \sqrt{s}} & 1\end{array}\right]\left[\begin{array}{cc}1 & s L \\ 0 & 1\end{array}\right]\left[\begin{array}{cc}0 & G \\ \frac{1}{G} & 0\end{array}\right]\left[\begin{array}{cc}1 & s m \\ 0 & 1\end{array}\right]\left[\begin{array}{c}F(\omega) \\ -U(\omega)\end{array}\right]$,

Where $L, K, G$, and $m$ are the inductance, the semi-inductance, the gyration coefficient, and the mass of the system respectively.

Let's isolating the $A B C D$ matrix part in Eq. 23 and setting $L, K, G$, and $m$ to be ' 1 ' for a simple to make the algebra simple calculation, the equation is reduced to

$\left[\begin{array}{cc}1 & 0 \\ 1 & 1 \\ \sqrt{s} & 1\end{array}\right]\left[\begin{array}{ll}1 & s \\ 0 & 1\end{array}\right]\left[\begin{array}{ll}0 & 1 \\ 1 & 0\end{array}\right]\left[\begin{array}{ll}1 & s \\ 0 & 1\end{array}\right]=\left[\begin{array}{cc}1 & s \\ 1 & \frac{s}{\sqrt{s}}+1\end{array}\right]\left[\begin{array}{ll}0 & 1 \\ 1 & s\end{array}\right]$.

Finally, the $A B C D$ matrix of the system in Figure 4 is

$\left[\begin{array}{l}\Phi(\omega) \\ I(\omega)\end{array}\right]=\left[T_{1}\right]\left[\begin{array}{c}F(\omega) \\ -U(\omega)\end{array}\right]=\left[\begin{array}{cc}A(s) & B(s) \\ C(s) & D(s)\end{array}\right]\left[\begin{array}{c}F(\omega) \\ -U(\omega)\end{array}\right]=\left[\begin{array}{cc}s & 1+s^{2} \\ \frac{s}{\sqrt{s}}+1 & \frac{1}{\sqrt{s}}+\frac{s^{2}}{\sqrt{s}}+s\end{array}\right]\left[\begin{array}{c}F(\omega) \\ -U(\omega)\end{array}\right]$,

Where $\Delta_{T 1}=-1$. Converting Eq. 25 into an impedance matrix,

$$
Z_{1}=\left[\begin{array}{ll}
Z_{11} & Z_{12} \\
Z_{21} & Z_{22}
\end{array}\right]
$$

Where

$$
\begin{aligned}
& Z_{11}=\frac{s}{\frac{s}{\sqrt{s}}+1}=\frac{s \sqrt{s}}{s+\sqrt{s}}(\equiv s \| \sqrt{s}), \\
& Z_{12}=\frac{-1}{\frac{s}{\sqrt{s}}+1}=-\frac{\sqrt{s}}{s+\sqrt{s}}, \\
& Z_{21}=\frac{1}{\frac{s}{\sqrt{s}}+1}=\frac{\sqrt{s}}{s+\sqrt{s}}, \\
& Z_{22}=\frac{\frac{1}{\sqrt{s}}+\frac{s^{2}}{\sqrt{s}}+s}{\frac{s}{\sqrt{s}}+1}=\frac{1+s^{2}+s \sqrt{s}}{s+\sqrt{s}} .
\end{aligned}
$$

By substituting ' $s$ ' with ' $j \omega$ ' one can easily find that all impedances of this system (Eq. 27, 28, 29 , and 30) are complex quantities, meaning that all have both real and imaginary parts in each frequency point. The results shown in Eq. 27 - Eq. 30 are a counter example that does not follow the traditional approach of a lossless LC network. In the other words, a lossy network has been realized without having a resistor in a system. We will show in the next section that this is due to existence of the semi-inductor in a system by comparing a case where the semi-inductor does not exist.

Using Eq. $11, Z_{\text {mot }}$ of this system can be calculated as

$Z_{\text {mot } 1}=\frac{1}{\left(\frac{s}{\sqrt{s}}+1\right)\left(\frac{1}{\sqrt{s}}+\frac{s^{2}}{\sqrt{s}}+s\right)}=\frac{s}{\sqrt{s}+s+s^{2}+2 s^{2} \sqrt{s}+s^{3}}$.

For computational benefits, we can convert Eq. 31 to an admittance $\left(Y_{\text {mot }}\right)$ to investigate the real part of $Z_{\text {mot' }}$

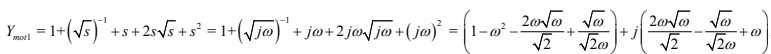

Since $\omega$ is always greater than 0 , the real part of Eq. 32 can have negative real parts if the equation satisfies

$$
\left(1-\omega^{2}-\frac{2 \omega \sqrt{\omega}}{\sqrt{2}}+\frac{\sqrt{\omega}}{\sqrt{2} \omega}\right)<0 .
$$

For example, if we have an angular frequency $\omega=1[\mathrm{rad} / \mathrm{sec}]$, Eq. 33 is satisfied $\left(1-1-\sqrt{2}-\frac{\sqrt{2}}{2}=-\frac{1}{\sqrt{2}}<0\right)$. We can generalize if $Y_{\text {mot }}$ is none positive then $Z_{\text {mot }}$ is also not positive. In this specific example, any angular frequency $(\omega)$ which satisfies Eq. 33 can have 

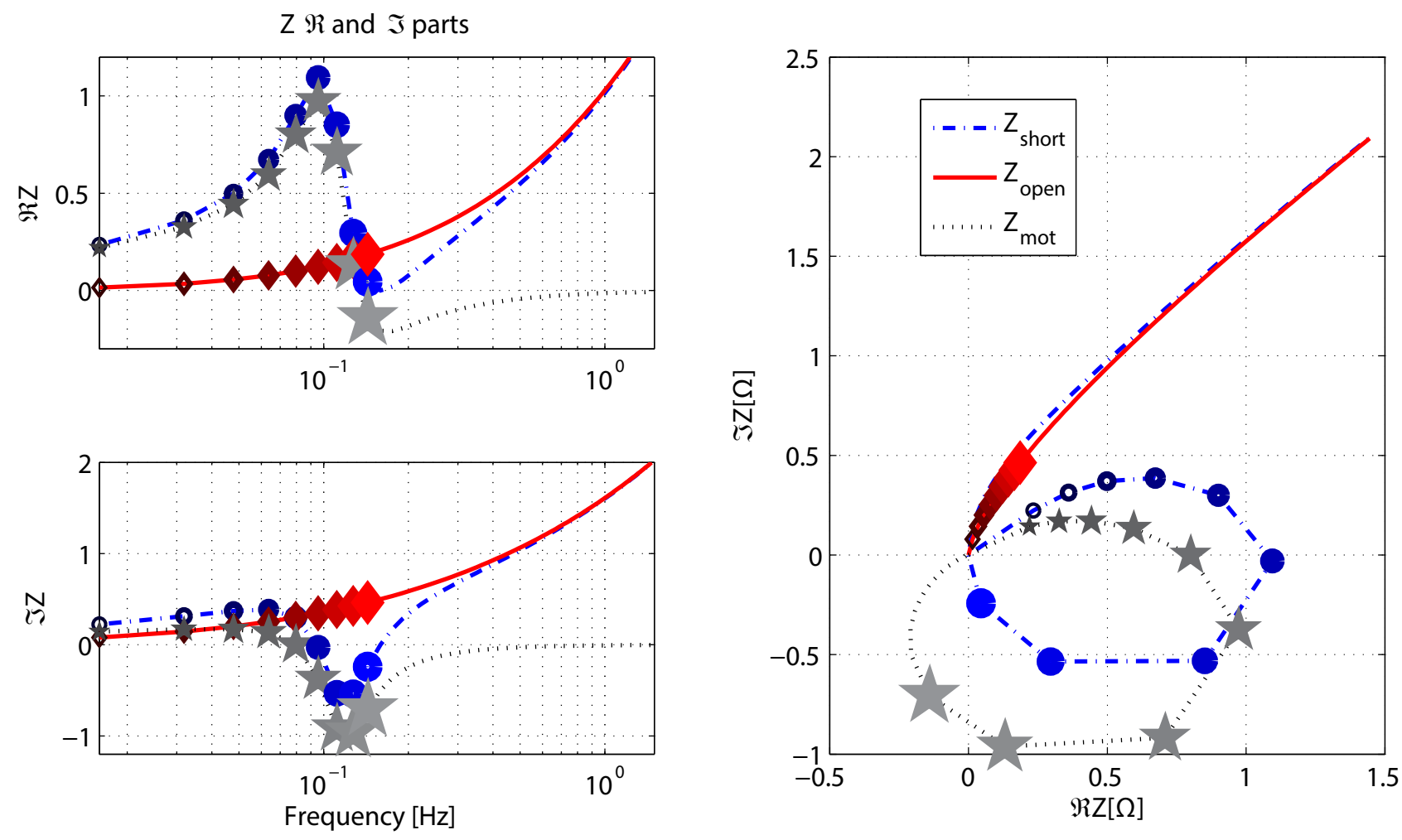

Figure 5: Computed motional impedance (Eq. 31), and input impedances with both open (Eq. 27) and short circuit conditions (Eq. $31+$ Eq. 27) based on a simple electro-mechanic network shown in Figure 4. The left figures show the real and imaginary parts of a simple electro-mechanic network. The marker's size indicates increment in frequency. Between $8^{\text {th }}$ and $9^{\text {th }}$ frequency points, the real parts of $Z_{\text {mot }}$ goes to negative.
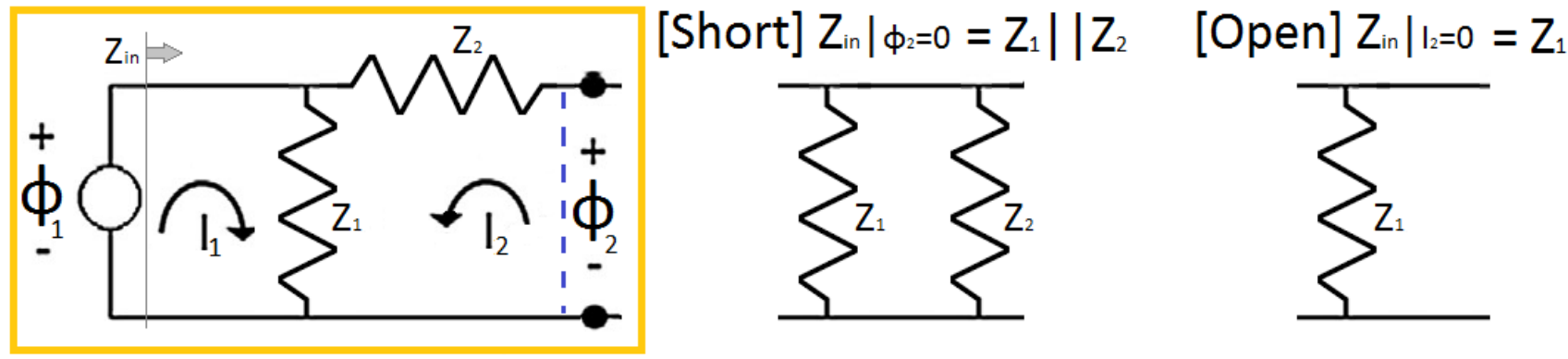

Figure 6: Demonstration of $Z_{\text {mot }}$ 's negative real part using a simple circuit example.

negative resistance in $Z_{\text {mot }}$. This $Z_{\text {mot }}$ is not a positive definite quantity, which means it does not conserve energy of the network. This mathematically supports that it is legal to have negative real parts in $Z_{\text {mot }}$ which is caused by the square root components in the system such as eddy-current.

Figure 5 shows the motional impedance and input impedances with both open and short circuit conditions. To help understand better, one can think the open circuit impedance when a system is de- magnetized, and the short circuit condition is the system's (i.e., a transducer) free oscillation in vacuum.

\section{Discussion}

In this section, we discuss interesting features of the $Z_{\text {mot }}$ its circular shape (in the complex plane) and investigate the reason to make it a circle. Based on the claim that we made, the negative real part of the $Z_{\text {mot }}$ is due to the diffusion of the mate- 
(1)

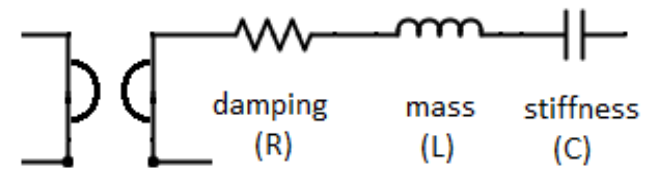

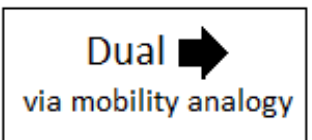

(2)

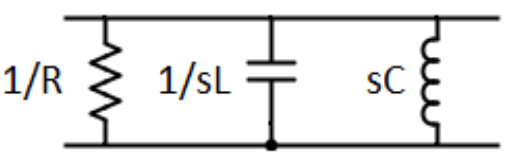

Figure 7: Due to the gyrator, the mechanical components become dual when they are seen on the electrical side of the network. The paralleled resistor, capacitor and inductor network contributes to the circular shape of the $Z_{\text {mot }}$ polar plot.

Input impedance with mechanical damping $(\mathrm{R})$, mass $(\mathrm{L})$, stiffness $(\mathrm{C})$
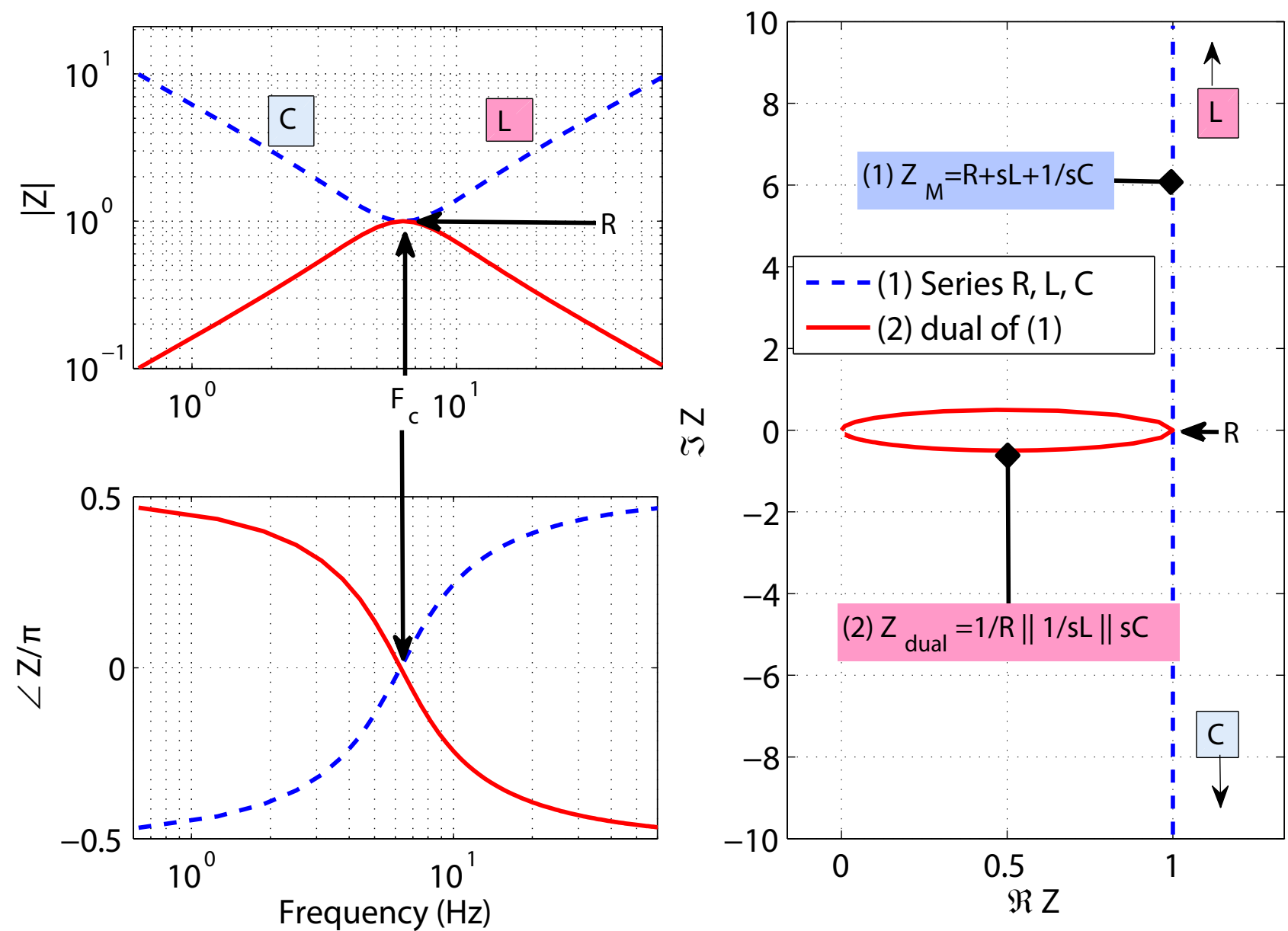

Figure 8: This figure explains the circular shape of $Z_{\text {mot }}$ where the motion of the mechanical behavior (i.e., damping (loss), mass, and stiffness) projected to the electrical side defines $Z_{\text {mot }}$. The blue line shows input impedance based on the series relationship ((1) in Figure 7 without considering the gyrator) while the red line represents the dual. The upper-left and lower-left plots show magnitude and phase of impedance and the right plot (polar plot) shows real and imaginary parts of the impedance. The red circle on the polar plot shows circular shape of $Z_{\text {mot }} . F_{c}$ stands for the transition frequency between $C$ (low-frequency) and $L$ (high-frequency). In polar plots, if $I Z \rightarrow+\infty, Z$ is dominated by $L$, and in case of $I Z \rightarrow-\infty, Z$ depends on $C$. Note that this figure only discusses the shape of typical $Z_{\text {mot' }}$, not its negative real parts. For simplification, values for $L, R$, and $C$, are 1 in this simulation.

rial, we briefly discuss the two kinds transformer's core losses via the basic electro-magnetic theories including the eddy current losses.

\section{The negative real parts of $\boldsymbol{Z}_{\text {mot }}$}

Figure 6 demonstrates a case where a difference of two input impedances $\left(Z_{i n}\right.$ with different 
boundary conditions) goes negative. For example, taking $Z_{1}=Z_{2}=100 \Omega$. Based on the definition of $Z_{\text {mot }}$ (Eq. 11), sub-tracting the open circuit impedance from the short circuit impedance results in $-50 \Omega\left(\left.Z_{\text {in }}\right|_{\Phi 2=0}-\left.Z_{\text {in }}\right|_{12}=0=Z_{1}|| Z_{2}-Z_{1}=50 \Omega-100 \Omega\right)$. This simplest example tells us a lot about the nature of $Z_{\text {mot' }}$ as well as modeling the electro-mechanic system.

Next consider an electro-mechanic system. If there is no shunt resistance (i.e., $Z_{1}$ ) in the electrical side of the system, $Z_{\text {mot }}$ cannot have negative real part as we see in Figure 6. Current is split at the parallel junction. Once the divided partial current flows through the shunted component(s) to ground, the other components (across the parallel junction), cannot see the split current. The other components are not affected by this split current which does not flow inside them. Therefore, the current is loss as the other components point of view.

To apply this theory to our system explaining the negative real parts of $Z_{\text {mot }^{\prime}}$ the one possible physical place for the shunt component loss is the eddy current, the diffusing current into magnetic core such as armature in our specific case. It has been shown experimentally since Kennelly and Pierce [19], that $Z_{\text {mot }}$ has negative real parts. This fact supports the view that a shunt loss in electrical side of the system must contribute to this loss (semi-inductor) when modeling the system [16].

\section{The circular shape of $Z_{\text {mot }}$}

In the polar impedance plane (R vs. I), $Z_{\text {mot }}$ is a circle passing through the origin [20]. The unusual shape may be explained by the physical nature of the anti-reciprocal electromechanic systems. The left side circuit (1) in Figure 7 describes a (typical) mechanical electromechanic network. The series of a damper, a mass, and a stiffness of the system are represented as circuit components $R, L$, and $C$, respectively. The $Z_{\text {mot }}$ is defined as a mechanical characteristic observed on the electrical side; therefore, simulation of these three main mechanical elements on the electrical side is our main concern.

Two circuits shown in Figure 7 are functionally equivalent. Part (1) is physically intuitive due to using a gyrator, and (2) is a dual version of (1) via the mobility analogy [21]. Figure 8 simulates the two circuit cases in Figure 7; the blue line (1) Without the gyrator (purely mechanical case) and the red line (2) Decoding the gyrator using mobility method to see the mechanical behavior on the electrical input side. The upper and lower plots in the left plane represent the magnitude and phase of the input impedance, and the right polar plot shows the real and imaginary parts of the impedance.

In Figure 8 , the red circle on the polar plot $\left(Z_{\text {dual }}\right)$ shows $Z_{\text {mot }}$, which is the dual of ZM namely,

$$
\begin{aligned}
& Z_{M}=R+\frac{1}{s C}+\left.s L\right|_{R, L, C=1}=1+\frac{1}{j \omega}+j \omega=\left\{\begin{array}{cc}
\infty & \omega \rightarrow \infty \\
1 & \omega \rightarrow 1 \\
-\infty & \omega \rightarrow-\infty
\end{array},\right. \\
& Z_{\text {dual }}=\frac{1}{R} \|\left. s C||_{s L}\right|_{R, L, C=1}=\frac{1}{1+j \omega+\frac{1}{j \omega}}=\left\{\begin{array}{cc}
0 & \omega \rightarrow 0 \\
1 & \omega \rightarrow 1 \\
0 & \omega \rightarrow \infty
\end{array} .\right.
\end{aligned}
$$

The reason we have a circle shape of $Z_{\text {mot }}$ is because we are observing mechanical behavior across the gyrator. Note that $F_{c}$ stands for the transition frequency between $C$ (low-frequency) and $\mathrm{L}$ (high-frequency) for both original and dual of magnitude and phase plots. In polar plots, when $I Z \rightarrow$ $+\infty, Z$ is dominated by $L$ and in case of $I Z \rightarrow-\infty, Z$ depends on $C$.

One may suggest a refined model of $Z_{\text {mot }}$ based on Figure 7 . The only difference between the real experimental data of $Z_{\text {mot }}$ and the simulation in Figure 8 is the angular rotation (clockwise direction) of the circle pivoted at the origin of the circle, which will introduce the negative real part in $Z_{\text {mot }}$. One way to realize this model is to add a phase delay in the system $\left(e^{-j \varphi(\omega)}\right)$ along with mechanical circuits.

Rotating the circle toward the negative real part is related to any shunt loss in the electrical part of the system.

\section{Conclusions}

It is interesting to review history of impedance concept in retroactive manner. Study on impedance was built over a long-time span from Ohm to Brune. Since the AC impedance concept was developed by Kennelly [2], it took 28 years to rigorously define the properties of the impedance by Brune [22].

In this study, anti-reciprocity, a unique two port network's postulate, has been investigated via a specific real-world example, the BAR model. $Z_{\text {mot }}$ has been simulated and its result has demonstrated that it is not a physically realizable PR impedance with respect to Brune's impedance postulates [22]. In this sense, $Z_{\text {mot }}$ is a transfer impedance not a driv- 
ing point impedance. Moreover, we have studied the physics behind of $Z_{\text {mot }}$ 's non-PR characteristic which lies in one of the core losses embedded in an EM system; the Eddy current loss (a theoretically appreciated shunt loss in electrical side of the system) which is independent of the coupling between the current and the force in the system. The $Z_{\text {mot }}$ reflects a purely mechanical behavior, a resonance, when it is looked at electrical side, thus it has a circular shape. And we have shown that the nature of anti-reciprocity makes a loop in the impedance of the electro-mechanic system by applying the mobility analogy with a gyrator which represents an anti-reciprocal characteristic. In summary, one of the most unique anti-reciprocal system characteristic, $Z_{\text {mot }}$ has been fully and uniquely analyzed based on both the empirical and theoretical foundations for the first time since its invention by A.E. Kennelly in 1912.

\section{References}

1. S Ramo, JR Whinnery, T Van Duzer (1965) Fields and waves in communication electronics. Wiley, New York, USA.

2. E Kennelly (1893) Impedance. Transactions of the American Institute of Electrical Engineers 10: 172232.

3. O Heaviside (1892) On resistance and conductance operators, and their derivatives inductance and permittance, especially in connection with electric and magnetic energy. Electrical Papers 2: 355-374.

4. GA Campbell (1903) XXX. on loaded lines in telephonic transmission. Philosophical Magazine Series 5: 313330.

5. ME Van Valkenburg (1964) Network analysis. Prentice-Hall, Englewood Cliffs, USA.

6. GA Campbell (1922) Physical theory of the electric wave-filter. Bell System Technical Journal 1: 1-32.

7. ME Van Valkenburg (1960) Introduction to modern network synthesis. Wiley, New York.

8. E Kennelly, H Nukiyama (1919) Electromagnetic theory of the telephone receiver with special reference to motional impedance. Proceedings of the American Institute of Electrical Engineers 38: 491-539.

9. E Kennelly, K Kurokawa (1921) Acoustic impedance and its measurement. Proceedings of the American Academy of Arts and Science 56: 3-42.

10.E Kennelly (1925) The measurement of acoustic impedance with the aid of the telephone receiver.
Journal of the Franklin Institute 200: 467-487.

11.RL Wegel (1921) Theory of magneto-mechanical systems as applied to telephone receivers and similar structures. Journal of the American Institute of Electrical Engineers 40: 791-802.

12.J Vanderkooy (1989) A model of loudspeaker driver impedance incorporating eddy currents in the pole structure. Journal of the Audio Engineering Society 37: 119-128.

13. TS Littler (1934) Motional impedance diagrams. Journal of the Acoustical Society of America 5: 235-241.

14.RD Fay, WM Hall (1933) The determination of the acoustical output of a telephone receiver from input measurements. Journal of the Acoustical Society of America 5: 46-56.

15.CR Hanna (1925) Design of telephone receivers for loud speaking purposes. Proceedings of the Institute of Radio Engineers 13: 437-460.

16.N Kim, JB Allen (2013) Two-port network analysis and modeling of a balanced armature receiver. Hear Res 301: 156-167.

17.FV Hunt (1954) Electroacoustics: The analysis of transduction and its historical background. Harvard University Press, Cambridge, USA.

18.J Jensen, FT Agerkvist, JM Harte (2011) Nonlinear time-domain modeling of balanced-armature receivers. Journal of the Audio Engineering Society 59: 91101.

19.E Kennelly, GW Pierce (1912) The impedance of telephone receivers as affected by the motion of their diaphragms. Proceedings of the American Academy of Arts and Science 48: 113-151.

20.E Kennelly, HA Affel (1915) The mechanics of telephone-receiver diaphragms, as derived from their motional impedance circles. Proceedings of the American Academy of Arts and Science 51: 421-482.

21.FA Firestone (1938) The mobility method of computing the vibration of linear mechanical and acoustical systems. Journal of the Acoustical Society of America 10: 83-83.

22.0 Brune (1931) Synthesis of a finite two-terminal network whose driving-point impedance is a prescribed function of frequency. Massachusetts Institute of Technology, USA. 